\title{
Dopamine drives the circuit
}

Flies rely on their sense of smell to navigate their environment. Mushroom body neurons have a key role in olfactory memory, but little is known about the circuits that provide these cells with relevant information. Two studies now identify distinct dopaminergic projections to specific mushroom body neurons that regulate different aspects of olfactory learning and memory in Drosophila melanogaster.

Miesenböck and colleagues used a training paradigm in which a fly learned to associate one of two odours with an electric shock; as a result, the fly avoided that odour in subsequent trials. The authors showed that such an olfactory memory could also be induced by pairing exposure to an odour with activation - using an optogenetic activation system - of dopaminergic neurons. This suggested that dopamine signalling mediates the storage of aversive odour associations.

Using the same "remote control" to target smaller groups of dopaminergic neurons revealed that a particular set of four dopaminergic cell clusters was required for aversive olfactory learning; indeed, blocking activity in these clusters prevented learning. By labelling the neurons in these clusters the authors showed that only the paired posterior lateral cluster 1 (PPL1) projects to the mushroom bodies - specifically, to the vertical and horizontal lobes; this identifies these 12 cells (or a smaller subset of them) as the source of the reinforcement signal for aversive olfactory conditioning.

Waddell and colleagues investigated how appetitive olfactory memory retrieval is promoted by hunger. They used a reinforcement learning paradigm in which flies learned to associate one of two odours with a sugar reward. The flies developed a memory for the conditioned odour, but this memory was expressed (as a behavioural preference for the odour) only in flies that were starved before testing. So how do states of hunger and satiety influence memory performance?

The authors focused on neuropeptide $\mathrm{F}$ (NPF), the D. melanogaster orthologue of mammalian neuropeptide $\mathrm{Y}$, which is an important regulator of feeding behaviour. Stimulating NPF neurons in the protocerebrum (by expressing a heat-sensitive transgene under the control of the transcriptional activator GAL4 driven by the NPF promoter and then increasing the ambient temperature) induced memory retrieval even in satiated flies. This suggests that satiation prevents memory retrieval by suppressing NPF signalling.
To identify the targets of NPF the authors used different GAL4 lines to drive transcription of small interfering RNA directed against the NPF receptor in different groups of neurons. Only food-deprived flies expressing the GAL4-c061 driver showed reduced olfactory memory. The six neurons expressing GAL4-c061 were found to be dopaminergic and to innervate the mushroom bodies specifically the medial lobe and peduncle — indicating that they are a subset of PPL1 neurons. Blocking the output of these neurons improved memory performance in satiated flies, whereas activating them inhibited memory performance in food-deprived flies. These findings identify a circuit that regulates memory performance in accordance with a fly's satiety state: NPF neurons inhibit dopaminergic PPL1 neurons, thereby removing the inhibitory input to mushroom body neurons and promoting olfactory memory.

These studies point to an olfactory memory circuit in D. melanogaster in which dopaminergic PPL1 neurons drive mushroom body neurons in a manner specific to the stimulus (rewarding or aversive) and the animal's motivational state (satiated or hungry).

Leonie Welberg

ORIGINAL RESEARCH PAPERS Claridge-Chang, A. et al. Writing memories with light-addressable reinforcement circuitry. Cell 139, 405-415 (2009) | Krashes, M. J. et al. A neural circuit mechanism integrating motivational state with memory expression in Drosophila. Cell 139, 416-427 (2009)

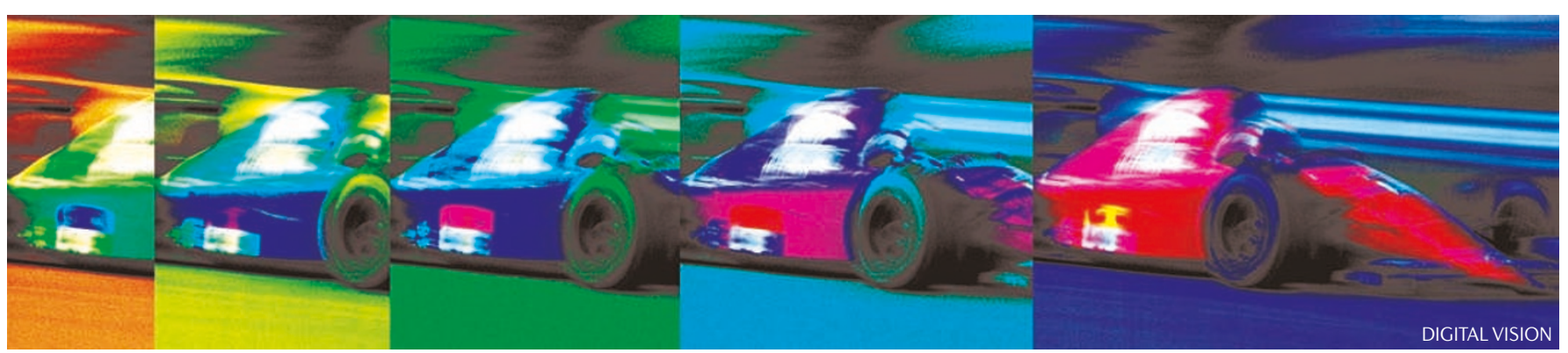

